\title{
SEARCH FOR HEAVY SEQUENTIAL NEUTRINOS AT PEP AND PETRA
}

\author{
R. THUN \\ Randall L.aboratory of Physics, University of Michigan, Ann Arbor, MI 48109, USA
}

Received 18 October 1983

\begin{abstract}
The experimental signature of the production of heavy, sequential neutrinos at the PFP and PFTRA $\mathrm{e}^{+} \mathrm{e}^{-}$colliders is discussed. The model for this discussion assumes that in analogy with quarks the neutrino mass spectrum rises sharply and that massive neutrinos decay via charged currents involving as yet unknown mixing parameters.
\end{abstract}

1. Introduction. The observed pattern of lepton and quark doublets continues to elude theoretical understanding. This "generation" problem has been addressed by either enlarging symmetry groups to include all leptons and quarks within fundamental representations [1] or by treating these particles as composite structures [2]. Neither approach has yielded any substantial insight into the observed fermion spectrum.

The most fundamental parameter to be determined is the number of generations. Searches at PETRA and PEP have yielded no new charged leptons or quarks beyond the third-generation $\tau$ and $b$ [3]. Searches are presently underway at the CERN $\bar{p}$ collider for the $t$ quark and possible fourth-generation charged leptons and charged $1 / 3$ quarks. These searches are limited strictly by the available energy of $30-40 \mathrm{GeV}$ at the $\mathrm{e}^{+} e^{-}$colliders and essentially by the observed masses of the $W$ and $Z$ bosons at the $\bar{p} p$ collider. These limitations are particularly severe since the masses of the observed charged leptons and quarks increase rapidly with generation number.

Perhaps the question of the number of generations is best approached in the neutrino sector. At least two possibilities exist. The first is that all neutrinos are light compared to the $Z^{0}$ mass in which case precision measurements of the $Z^{0}$ width or measurements of $\mathrm{e}^{+} \mathrm{e}^{-} \rightarrow \gamma \bar{\nu} \nu$ will yield a definitive answer [4]. A second possibility, and the one addressed here, is that the neutrino spectrum starts with a low mass but rises rapidly as is the case for the other fermions. Such a possible spectrum, consistent with known neutrino mass bounds, is sketched in fig. 1. If this picture is correct, then new and as yet unexploited opportunities exist for discovering higher generations by observing the decay of unstable, heavy neutrinos. Indeed, the PEP and PETRA colliders have sufficient energy and luminosity to produce neutrino pairs by the process $\mathrm{e}^{+} \mathrm{e}^{-} \rightarrow$

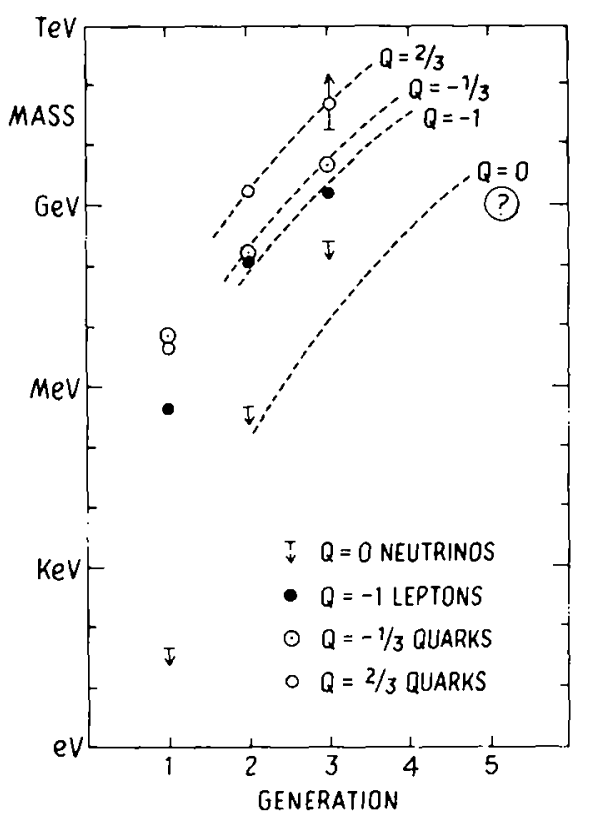

Fig. 1. Mass spectrum for quarks and leptons. For neutrinos only mass limits are shown. The curves are all hypothetical. 
$\mathrm{Z}^{0} \rightarrow \bar{\nu} \nu$ at a rate that exceeds 30 per year for each interaction region. What follows is a discussion of the possible signals for the production of heavy, unstable neutrinos at these two $\mathrm{e}^{+} \mathrm{e}^{-}$colliders. It is assumed that the standard $S U(2) \times U(1)$ electroweak theory describes both the production and decay of such neutrinos. A search for heavy electron neutrinos in $\mathrm{e}^{+} \mathrm{e}^{-} \rightarrow \mathrm{E}^{0_{\bar{\nu}}}$ has been described elsewhere [5]. Experimental limits on the production of heavy neutral leptons have also been obtained in muon and neutrino interactions [6].

2. Production of massive neutrinos in $e^{+} e^{-}$annihilations. If the coupling of heavy neutrinos to electrons is small, then the production of such neutrinos will occur primarily through the $Z^{0}$ in termediate state (fig. 2a) and $W$ exchange (fig. 2b) may be neglected. Assuming no initial-state polarization and averaging over final state spins, the differential cross section [7] for $\mathrm{e}^{+} \mathrm{e}^{-} \rightarrow \bar{\nu} \nu$ is:

$$
\begin{aligned}
& \mathrm{d} \sigma / \mathrm{d} \Omega=\left\{\alpha^{2} s /\left[\left(s-m_{Z}^{2}\right)^{2}+m_{Z}^{2} \Gamma_{Z}^{2}\right]\right\} \\
& \quad \times\left[\frac{1}{4}\left(\left|a_{\mathrm{e}}\right|^{2}+\left|b_{\mathrm{e}}\right|^{2}\right)\left(\left|a_{\nu}\right|^{2}+\left|b_{\nu}\right|^{2}\right)\left(1+\cos ^{2} \theta\right)\right. \\
& \left.\quad+2 \operatorname{Re}\left(a_{\mathrm{e}} b_{\mathrm{e}}^{*}\right) \operatorname{Re}\left(a_{\nu} b_{\nu}^{*}\right) \cos \theta\right],
\end{aligned}
$$

(a)

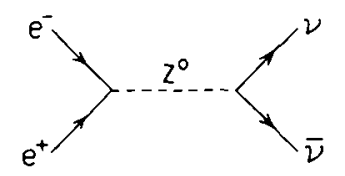

(b)

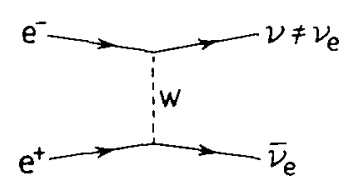

(c)

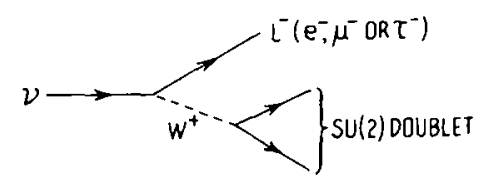

Fig. 2. (a) and (b) Possible mechanisms for producing heavy, sequential neutrinos in $\mathrm{e}^{+} \mathrm{e}^{-}$interactions. The $\mathrm{W}$-exchange process is assumed to be negligible. (c) Decay of a heavy neutrino. where $\alpha$ is the fine structure constant, $s$ the $\mathrm{E}_{\mathrm{c} . \mathrm{m}}^{2}$., $m_{\mathrm{Z}}$ the $\mathrm{Z}^{0}$ mass, $\Gamma_{\mathrm{Z}}$ the $\mathrm{Z}^{0}$ width,

$a_{\mathfrak{c}}=\left(-1+4 \sin ^{2} \theta_{w}\right) / 2 \sin 2 \theta_{w}, \quad b_{\mathfrak{c}}=-1 / 2 \sin 20_{w}$,

$a_{\nu}=b_{\nu}=1 / 2 \sin 2 \theta_{\mathrm{w}}$,

and $\theta$ is the scattering angle between $\mathrm{e}^{+}$and $\bar{\nu}$.

For $m_{\mathrm{Z}}=93 \mathrm{GeV}, \Gamma_{\mathrm{Z}}=2.8 \mathrm{GeV}$ and $\sin ^{2} \theta_{\mathrm{w}}=$ 0.23 one obtains at the PEP energy of $29 \mathrm{GeV}$ :

$$
\begin{aligned}
\mathrm{d} \sigma / \mathrm{d} \Omega & =\left(2.8 \times 10^{-37} \mathrm{~cm}^{2}\right) \\
& \times\left[0.063\left(1+\cos ^{2} \theta\right)+0.020 \cos \theta\right]
\end{aligned}
$$

and the integrated cross section is

$\sigma=\frac{4}{3} \pi \alpha^{2} \frac{0.25 s}{\left(s-m_{Z}^{2}\right)^{2}+m_{Z}^{2} \Gamma_{Z}^{2}}=3.0 \times 10^{-37} \mathrm{~cm}^{2}$.

At present PEP delivers a yearly in tegrated luminosity of about $10^{38} \mathrm{~cm}^{-2}$ yielding approximately 30 events for the above cross section. It is therefore feasible to search for heavy neutrinos at a storage ring such as PEP or PETRA.

3. Decays of heavy neutrinos. In analogy with charge $-1 / 3$ quark decays, heavy neu trinos are assumed to decay to the most massive charged lepton allowed by energy conservation plus a charged virtual $\mathrm{W}$ which couples to a lepton or quark doublet (e $\mathrm{e}, \mu \nu$, $\mathrm{u} \overline{\mathrm{d}}$, etc.) as shown in fig. $2 \mathrm{c}$. The only unknown parameters in specifying the decay properties within the standard weak interaction theory are the mass of neutrino, $M$, and the mixing angle describing the coupling of the massive neutrino to the charged lepton. If the decay products of the heavy neutrino are all light, then the lifetine of the neutrino is given by

$t_{\nu} \approx\left(1 / \epsilon N_{W}\right)\left(m_{\mu} / M\right)^{5} t_{\mu}$,

where $m_{\mu}=0.106 \mathrm{GeV}$ is the muon mass, $t_{\mu}=2.2 \times$ $10^{-6} \mathrm{~s}$ the muon lifetime, $M$ the mass of $\nu, N_{W}$ the number of fundamental fermion doublets that are kinematically allowed to couple to the virtual $W$. $\epsilon \leqslant 1$ describes the mixing mentioned above.

When one of the decay products has a non-negligible mass $m$, the life time given in (4) increases by a factor [8] $f=\left(1-8 y+8 y^{3}-y^{4}-12 y^{2} \ln y\right)^{-1}$ where $y=m^{2} / M^{2}$.

The requirement that the decay be observable in a 
(a)

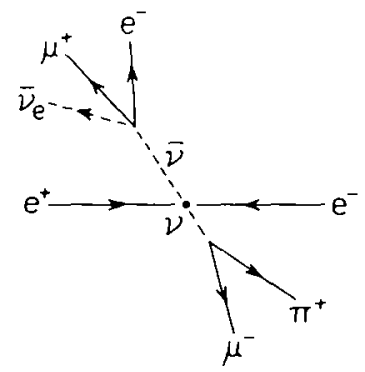

(b)

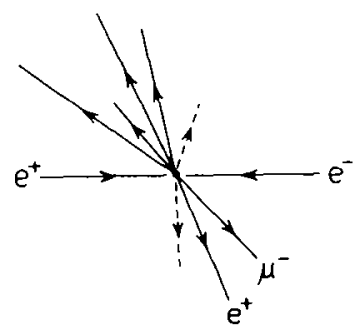

Fig. 3. Possible event topologies for the production and decay of heavy neutrinos in $\mathrm{e}^{+} \mathrm{e}^{-}$annihilations. In (a) it is assumed that decay vertices are resolved whereas this is not the case in (b).

detector places a lower limit on the accessible mass region:

$\gamma c t=\left(E_{0} / M c^{2}\right)\left(c / \epsilon N_{\mathrm{W}}\right)\left(m_{\mu} / M\right)^{5} t_{\mu} \leqslant 1 \mathrm{~m}$.

For a beam energy $E_{0}=14.5 \mathrm{GeV}$ and assuming $\epsilon=1$ and $N_{\mathrm{W}}=5$ (for e $\left.\nu+\mu \nu+3 \mathrm{u} \overline{\mathrm{d}}\right)$ one obtains:

$M \geq 0.5 \mathrm{GeV}$.

This mass limit may be significantly higher if $\epsilon \ll 1$. (For example, $\epsilon=0.04$ yields $M \geq 0.9 \mathrm{GeV}$.)

If $M<m_{\tau}$ the possible decay products of heavy neutrinos are similar to those from the $\tau$ lepton except for the interchange $\nu_{\tau} \rightarrow \mu$. One expects:

$$
\begin{aligned}
\nu \rightarrow \mu^{-} \mathrm{e}^{+} \nu_{\mathrm{e}} & \approx 20 \%, \\
\rightarrow \mu^{-} \mu^{+} \nu_{\mu} & \approx 20 \%, \\
\rightarrow \mu^{-} \text {hadrons } & \approx 60 \% .
\end{aligned}
$$

The last category includes fully reconstructable modes such as $\mu^{-} \pi^{+}(210 \%)$.

For $m_{\tau}<M \lessgtr 5 \mathrm{GeV}$ one obtains:

$$
\begin{aligned}
\nu \rightarrow \tau^{-} \mathrm{e}^{+} \nu & \approx 20 \%, \\
\rightarrow \tau^{-} \mu^{+} \nu & \approx 20 \%, \\
\rightarrow \tau^{-} \text {hadrons } & \approx 60 \% .
\end{aligned}
$$

None of these modes are fully reconstructable since the $\tau$ decays into final states containing a neu trino. For neutrino masses below $\approx 5 \mathrm{GeV}$ the majority of $\mathrm{e}^{+} \mathrm{e}^{-} \rightarrow \bar{\nu} \nu$ events would have low charged multiplicities $\left(n_{\mathrm{ch}}=4\right.$ or 6$)$.

4. Signatures for heavy neutrinos in $e^{+} e^{-}$annihilations. From the above considerations, the production of heavy neutrinos may exhibit some or all of the following characteristics:

(i) Distinct decay vertices with tracks whose charges sum to zero.

(ii) Unusual lepton combinations in the final state.

(iii) Missing energy and unbalanced momentum associated with unobserved final-state neutrinos.

(iv) Low multiplicities.

These characteristics are illustrated in fig. 3a which displays an event of the type $\mathrm{e}^{+} \mathrm{e}^{-} \rightarrow\left(\bar{\nu} \rightarrow \mu^{+} \mathrm{e}^{-} \nu_{\mathrm{c}}\right)$ $\left(\nu \rightarrow \mu^{-} \pi^{+}\right)$. The most compelling evidence for neutral leptons would involve the observation of distinct decay vertices. Expression (4) with $N_{\mathrm{W}}=5$ gives

$\gamma c t \approx 2.6 \mathrm{~cm} / \epsilon M^{6}$,

where the mass $M$ of the heavy neutrino is given in $\mathrm{GeV}$. For mixing pararneters $\epsilon \ll 1$, decay paths of many centimeters are possible for neutrino masses in the interval from 1 to $\approx 3 \mathrm{GeV}$. The efficiency for observing such long-lived particles may be quite low in experiments that require in their trigger the passage of charged particles close to the beam pipe.

If the neutrinos are so massive and therefore shortlived that their decay vertices are no longer resolvable, then the search for their production becomes more difficult but not impossible. Backgrounds from higherorder QED processes such as $\mathrm{e}^{+} \mathrm{e}^{-} \rightarrow \mathrm{e}^{+} \mathrm{e}^{-} \tau^{+} \tau^{-}$and from low-multiplicity hadronic events containing semi-leptonic decays of $c$ or $b$ quarks share characteristics (ii)-(iv) listed above for events containing heavy unstable neutrinos. In this case one would search for unusual topologies such as illustrated in fig. $3 \mathrm{~b}$ where an event is shown which contains in one hemisphere an isolated $\mu$ e pair with an energy significantly below the beam energy. If events of this type are found, a 
careful study of backgrounds would be required.

Such a study is beyond the scope of this paper.

5. Conclusion. Searches for heavy, unstable, sequential neutrinos are feasible at PEP or PETRA. The most powerful signature for their existence would be the observation of distinct decay vertices in lowmultiplicity events. Other signatures involve unusual lepton combinations and missing energy or unbalanced momentum in the final state. Although a specific model for heavy neutrinos was considered in this paper, the enumeration of interesting event signatures applies to more general searches for new unstable neutral particles.

The author acknowledges helpful discussions with S. Errede, G. Kane, P. Tsai, and M. Veltman.

\section{References}

[1] Fxamples of applying group concepts to the generation problem include

F. Wilczek and A. Zee, Phys. Rev. Lett. 42 (1979) 421;

F. Fahri and L. Susskind, Phys. Rev. D20 (1979) 3404;

R.N. Cahn and H. Harari, Nucl. Phys B176 (1980) 135.

[2] Some recent composite models are described in

H. Terazawa, Y. Chikashige and K. Akama, Phys. Rev. D15 (1977) 480;

H. Harari, Phys. Lett. 86B (1979) 83.

[3] A review of these searches is given by S. Yamada, Proc. 1983 Intern. Symp. on Lepton and photon interactions at high energies, to be published.

[4] G. Barbiellini, B. Richter and J.L. Siegriest, Phys. Lett. 106B (1981) 414, and references therein.

[5] JADE-Collaboration, W. Bartel et al., Phys. Lett. 123B (1983) 353.

[6] A.R. Clark et al., Phys. Rev. Lett. 46 (1981) 299; CHARM-Collaboration, I. Bergsma et al., Phys. Lett. 128B (1983) 361.

[7] See, for example F.M. Renard, Basics of electron positron collisions (Fditions Frontieres, Dreux, 1981).

[8] Y.S. Tsai, Phys. Rev. D4 (1971) 2821. 